\title{
A Game-Theoretic Approach and Evaluation of Adversarial Vehicular Platooning
}

\author{
Imran Sajjad \\ Utah State University \\ 2400 Old Main Hill \\ Logan, Utah 84321 \\ imran.sajjad@aggiemail.usu.edu
}

\author{
Rajnikant Sharma \\ University of Cincinnati \\ 2600 Clifton Ave \\ Cincinnati, Ohio 45220, Ohio \\ rajnikant.sharma@uc.edu
}

\author{
Ryan Gerdes \\ Virginia Tech Research Center - \\ Arlington \\ 900 N Glebe Rd \\ Arlington, Virginia 22203 \\ rgerdes@vt.edu
}

\begin{abstract}
In this paper, we consider an attack on a string of automated vehicles, or platoons, from a game-theoretic standpoint. Game theory enables us to ask the question of optimality in an adversarial environment; what is the optimal strategy that an attacker can use to disrupt the operation of automated vehicles, considering that the defenders are also optimally trying to maintain normal operation. We formulate a zero-sum game and find optimal controllers for different game parameters. A platoon is then simulated and its closed loop stability is then evaluated in the presence of an optimal attack. It is shown that with the constraint of optimality, the attacker cannot significantly degrade the stability of a vehicle platoon in nominal cases. It is motivated that in order to have an optimal solution that is nearly unstable, the game has to be formulated almost unfairly in favor of the attacker.
\end{abstract}

\section{CCS CONCEPTS}

-Security and privacy $\rightarrow$ Security in hardware; Systems security; •Computer systems organization $\rightarrow$ Embedded and cyber-physical systems; Real-time systems;

\section{KEYWORDS}

Cyber-physical systems security, autonomous vehicles

\section{ACM Reference format:}

Imran Sajjad, Rajnikant Sharma, and Ryan Gerdes. 2016. A Game-Theoretic Approach and Evaluation of Adversarial Vehicular Platooning. In Proceedings of 2017 1st International Workshop on Safe Control of Connected and Autonomous Vehicles (SCAV 2017), Pittsburgh, PA USA, April 2017 (SCAV 2017), 7 pages.

DOI: http://dx.doi.org/10.1145/3055378.3055383

\section{INTRODUCTION}

Automated highway systems and self-driving cars are getting ever closer to real-world implementation. Multiple automated vehicles following each other on a highway with technologies like adaptive cruise control naturally form strings or platoons [31]. In this setting,

Permission to make digital or hard copies of all or part of this work for personal or classroom use is granted without fee provided that copies are not made or distributed for profit or commercial advantage and that copies bear this notice and the full citation on the first page. Copyrights for components of this work owned by others than ACM must be honored. Abstracting with credit is permitted. To copy otherwise, or republish, to post on servers or to redistribute to lists, requires prior specific permission and/or a fee. Request permissions from permissions@acm.org.

SCAV 2017, Pittsburgh, PA USA

(C) 2017 ACM. 978-1-4503-4976-5/17/04 . \$\$15.00

DOI: http://dx.doi.org/10.1145/3055378.3055383 the vehicles use some control law to adjust the distance between themselves and neighboring cars.

The automation of cars has shown to have numerous benefits including increased safety, passenger comfort and lower environmental impact $[1,25]$. They also have been shown to have better fuel efficiency [16] and to significantly reduce congestion on highways [24].

With this automation, new challenges and vulnerabilities surface. Platoons generally form a cyber-physical system (CPS) and there is a large body of work in this general area. With regards to platooning and automated vehicles, there is much work that relates directly to attack detection and identification $[10,17,20]$, much less work has been done to evaluate the efficacy of such attacks from the attacker's perspective. Chen et. al. use optimal control to find an attack in constrained conditions [6]. In [11], the authors attempt to find a generalized formulation of attacks based on system vulnerabilities. Ramasubramanian et. al. [23] investigate a system's resilience to attacks based on its structure.

With regards to platooning specifically, much work has been done on homogeneous vehicles and what criteria should be satisfied for acceptable operation and real-world deployment. Much work has been done evaluating the stability and string stability of a platoon [5, 21, 27, 29]. Most platooning involves a specific control law that is used to either regulate distance from the front (unidirectional) or from both front and back (bidirectional) [2, 32]. There are also possibilities for inter-vehicle communication that add a new layer of abstraction.

In [7], it is shown that a platoon is vulnerable to attacks if an attacker applies a destabilizing control input. This is achieved by the attacker modifying controller gains in order to cause instability. Only the gains in one vehicle need to be modified to achieve this. This sort of an attack is also presented in [8], where a group of colluding attackers cause string instability and increase traffic congestion.

It is a common theme in CPS work that a threat model is proposed and any reactionary measures such as detection or mitigation are then formulated around the characteristics of that attack. The gametheoretic approach provides an alternate setting more related to optimal control that can be used to find an attack that optimizes certain criteria in an adversarial setting. While optimal control tries to find a minimizing solution to a problem, game theory similarly involves finding a saddle-point or Nash Solution [18] to a problem, which is, in a sense, optimal for both the players.

Game theory has been used in the field of control systems for some time now. There are classic problems in game theory such as 
pursuit-evasion that have been adapted to modern control problems [13]. The area has also been used in the area of system design and disturbance rejection [3,30]. Game theory is also used in communications and designing data networks [12]. In [9], a set of worst-case safe initial conditions to avoid a two car collision are derived using a game-theoretic framework.

The main contribution of this paper is that it applies methods from game theory to the problem of vehicular platooning and illustrates the behavior of an attacker employing an optimal strategy (in a game-theoretic sense). We motivate the proposition that in a realistic setting where the attacker is also a real world actor, it is very hard to achieve an optimal solution in which the attacker is actually causing catastrophic collisions and/or destabilization.

The remaining paper is organized as follows. A brief introduction to certain concepts from game theory and a quick formulation of the zero-sum case is given in section II. In section III, a problem formulation specific to our system is presented, with the necessary assumptions and simplifications elucidated. Section IV and V provide some numerical and simulation results. Section VI discusses these results and section VII concludes this paper.

\subsection{Assumptions and Attacker Capabilities}

In order to permit a real-world solution, we state some assumptions. We formulate an infinite-horizon zero-sum differential game in section II, which naturally leads to static gains and requires full-state feedback. This can be accomplished through inter-vehicle communication. Each vehicle can apply an acceleration input. While this approach does seem centralized in a sense, there has been research on implementing such algorithms in a decentralized manner $[14,19]$.

We limit the discussion to one attacker in this paper. One of the imposed conditions on the method used to solve Riccati equations is that the resulting system be stable. This then leads to the restriction that the attacker can only degrade stability and not cause instability.

\section{GAME THEORY PRELIMINARIES}

Game theory provides a setting in which two or more players influence their respective scores by choosing different strategies [4]. Naturally, there are constraints on how different strategies affect the scores of each player, which are referred to as rules of the game. These three items-score, strategies and rules-are found in every well-formulated game. There are other extensions of this idea such as mixed-strategy and stochastic games but for our purposes, the above three ideas are sufficient.

There is also a distinction between static and dynamic games, which differ in the following sense: if a player is able to react after another player's decision as the game proceeds, the game is dynamic, otherwise it is static. Dynamic games have a sense of successive moves and thus a sense of progression. When extended to continuous-time systems, this naturally extends to the idea of time.

In most cases of game theory we are interested in the Nash and saddle-point solutions [4] and the strategies which produce them. These solutions have the property where one player cannot 'surely' gain anything by deviating from this strategy. Suppose two players are at a Nash equilibrium and have their respective scores. If one player decides to chance a move away from this point in order to gain some score, there exists a move (strategy) for the other player to make the first one lose more than his initial score. Hence it is in the best interests of the first player to stay at the Nash equilibrium.

Game theory applied to continuous-time control systems results in what are known as differential games [4]. In this case, the players have access to their specific control inputs (strategies) and the system dynamics form the constraints to the system (rules). When the question of control or optimality arises, naturally there is some notion of cost (scores).

Consider the system defined by the following state space description.

$$
\dot{x}=f(t, x, u)
$$

where $x \in \mathbb{R}^{r n}$ is the state vector, $u \in \mathbb{R}^{p}$ is the input vector. In the state vector, $n$ is the number of agents, while $r$ is the system order for each agent ${ }^{1}$. Usually the inputs are partitioned along respective players. Each player then chooses his or her control input from a space of allowable control inputs. Let us limit the discussion to two players and consider the following formulation

$$
\begin{aligned}
J_{1} & =\int_{0}^{T} L_{1}(x, u, t) d t+\Phi_{1}(x(T)) \\
J_{2} & =\int_{0}^{T} L_{2}(x, u, t) d t+\Phi_{2}(x(T)) \\
\dot{x} & =f(t, x, u)
\end{aligned}
$$

where $J_{i}$ is the total cost of player $i, L_{i}$ is an instantaneous cost and $\Phi_{i}$ is a terminal cost at time $T$. If we partition the vector $u$ into two vectors $u_{1}$ and $u_{2}$ (not the individual elements of $u$ ) each of which contains the control inputs for each player, we can deal with the effects of these two inputs separately, that is we can weight them in the cost functions separately. Both players want to minimize their respective costs $J_{1}$ and $J_{2}$. In our case, all the defending cars can be thought of as one player with multiple different control inputs, and all the attackers can be thought of as the other player ${ }^{2}$.

One very useful simplification which we will make is that we restrict the game to be zero sum. This means that the equality $J_{1}+$ $J_{2}=0$ always holds. Zero-sum games are a good starting point in many game-theoretic approaches since they imply that the players are completely non cooperative. This imposes some important restrictions on the formulation and implies certain properties of the solution itself. For example, all solutions to non-cooperative zero-sum games will be saddle points [4].

Another very useful class of games are linear quadratic games. This sort of a formulation is similar to the linear quadratic regulator (LQR) and the construction of its solution also follows a similar process [28]. The functions $L_{i}$ are replaced with the quadratic forms and the system is restricted to be linear time invariant (LTI)

\footnotetext{
${ }^{1}$ This choice of state vector size is one which applies to the real system we will deal with in the next section. We present it now to avoid providing two conflicting formulations and avoiding confusion in vector sizes.

${ }^{2}$ 'Teams' might be a better word here, but 'player' is used throughout the literature.
} 


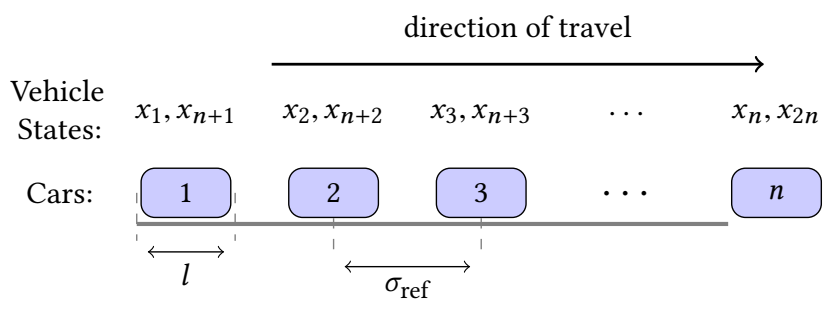

Figure 1: A Platoon of $n$ vehicles. Each car is $l$ meters long and the desired separation from center of one car to that of the other is $\sigma_{\text {ref. }}$ Car $n$ is the leader.

as shown in (3).

$$
\begin{aligned}
J_{1} & =\frac{1}{2} \int_{0}^{T} x^{T} Q_{1} x+u_{1}^{T} R_{11} u_{1}+u_{2}^{T} R_{12} u_{2} d t \\
& +\frac{1}{2} x^{T}(T) S_{T} x(T) \\
J_{2} & =\frac{1}{2} \int_{0}^{T} x^{T} Q_{2} x+u_{1}^{T} R_{21} u_{1}+u_{2}^{T} R_{22} u_{2} d t \\
& -\frac{1}{2} x^{T}(T) S_{T} x(T) \\
& Q_{1}+Q_{2}=0, R_{11}+R_{21}=0, R_{12}+R_{22}=0 \\
\dot{x} & =A x+B_{1} u_{1}+B_{2} u_{2}
\end{aligned}
$$

It is interesting to note at this point that the two cost functions are opposing each other. This captures the noncooperation and is absolutely necessary, since if both players have their goals aligned, a saddle point solution will not exist [4]. Also note that the values of $R_{12}$ and $R_{21}$ can be chosen to weight the other player's control input.

Without going into the details of constructing the solution, which can be found in $[4,28]$, the optimal control inputs $u_{1}^{*}$ and $u_{2}^{*}$ are given by

$$
\begin{aligned}
& u_{1}^{*}=-R_{11}^{-1} B_{1}^{T} P x \\
& u_{2}^{*}=R_{22}^{-1} B_{2}^{T} P x
\end{aligned}
$$

where $P$ is the solution to the following Ricatti Equation

$$
\dot{P}=-P A-A^{T} P-Q_{1}+P\left(B_{1} R_{11}^{-1} B_{1}^{T}-B_{2} R_{22}^{-1} B_{2}^{T}\right) P^{T}
$$

One further simplification which applies in our case of platooning is that we fix a terminal time of infinity (infinite horizon) and we evaluate the solution to (5) by setting the left hand side to zero.

\section{PROBLEM FORMULATION}

Consider the platoon of $n$ vehicles shown in Figure 1. The state vector is

$$
\begin{aligned}
& x=\left[\begin{array}{llllllll}
x_{1} & x_{2} & \ldots & x_{n} & x_{n+1} & x_{n+2} & \ldots & x_{2 n}
\end{array}\right]^{T}, \\
& u=\left[\begin{array}{llll}
u_{1} & u_{2} & \ldots & u_{n}
\end{array}\right]^{T}
\end{aligned}
$$

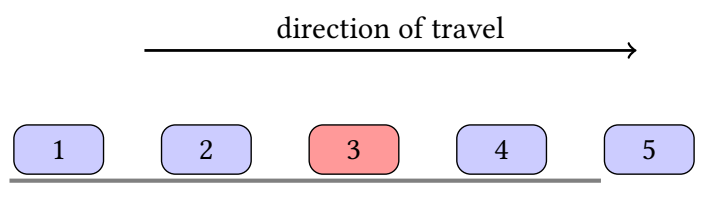

Figure 2: A Platoon of 5 vehicles with the attacker at 3.

and the complete LTI system is given by

$$
\begin{aligned}
& \dot{x}=A x+B u \\
& A=\left[\begin{array}{ll}
0_{n \times n} & I_{n \times n} \\
0_{n \times n} & 0_{n \times n}
\end{array}\right] \quad B=\left[\begin{array}{c}
0_{n \times n} \\
I_{n \times n}
\end{array}\right]
\end{aligned}
$$

where car $i$ has position and velocity $x_{i}, x_{n+i}$ respectively and control input $u_{i}$. These positions are measured from the center of mass of all the cars. Each car is essentially a double integrator in our setting. The choice of commanding acceleration directly is motivated by [22] where a split level control architecture is presented so that a higher level controller commands an acceleration and a lower level controller realizes it. A good portion of literature also assumes acceleration is commanded directly [2,32].

In order to have the system track a reference and have the cars maintain a separation, we define a state vector $z$ of error coordinates as follows

$$
\begin{array}{rlrl}
z_{1}=x_{2}-x_{1}-\sigma_{\text {ref }} & z_{n+1}=x_{n+2}-x_{n+1} \\
z_{2}=x_{3}-x_{2}-\sigma_{\text {ref }} & z_{n+2}=x_{n+3}-x_{n+2} \\
& \vdots & \vdots & \\
z_{n}=x_{\text {ref }}-x_{n} & z_{2 n}=\dot{x}_{\text {ref }}-x_{2 n}
\end{array}
$$

where $x_{\text {ref }}, \dot{x}_{\text {ref }}$ are the position and velocity of a reference trajectory for the leader. Under this transformation, the $A$ matrix remains the same, but the $B$ matrix becomes

$$
B^{\prime}=\left[\begin{array}{ccccc} 
& & 0_{n \times n} & & \\
-1 & 1 & 0 & \ldots & 0 \\
0 & -1 & 1 & \ldots & 0 \\
0 & 0 & -1 & \ldots & 0 \\
\vdots & \vdots & \vdots & \ddots & \vdots \\
0 & 0 & 0 & \ldots & -1
\end{array}\right]
$$

Considering the case where we have one attacker at position 3 in a 5 vehicle platoon (Figure 2), we can use the following parameters for the cost matrices in the game formulation.

$$
\begin{aligned}
& Q_{1}=\operatorname{diag}\left[\begin{array}{llllllllll}
1 & 1 & q_{3} & 1 & 1 & 2 & 2 & q_{8} & 2 & 2
\end{array}\right] \\
& R_{11}=\operatorname{diag}\left[\begin{array}{llll}
1 & 1 & 1 & 1
\end{array}\right] \\
& R_{22}=r_{2}
\end{aligned}
$$

where $q_{3}, q_{8}$ and $r_{2}$ are parameters which will be varied in the next section. In $Q_{1}$ the first half of the parameters weight position error and the second half velocity error. As mentioned before, the four cars except the attacker will constitute one player (the defender) and the single attacking car will be the other player.

Since the game is zero sum, we need not define the other three cost matrices explicitly. Also, since we formulated our problem in error coordinates which the platoon directly has to minimize, we can start off with diagonal cost matrices. The defenders want to 
minimize their own error states so their entries in the cost matrix are positive. In turn, the attacker would then want to maximize these error states. The variables $q_{3}$ and $q_{8}$ signify how much the attacker is willing to move in order to cause the other cars to move.

When solved with these values set, the result is two gain matrices (for the defenders and attackers) that use full state feedback for each control input. This was expected since we chose an infinite time horizon. But even with these gains, we can evaluate the closed loop stability of the system by looking at the pole locations and how they change with varying parameters.

As mentioned before some form of inter-vehicle communication will probably be required to realize full state feedback. Local sensing will probably prove to be insufficient. But readers might be interested in decentralized cooperative localization where individual agents estimate a full system state based on locally observed data with limited communication and possibly intermittent global data. $[19,26]$ provide some solutions on whether a full state estimator is possible or not using only local sensing.

\section{GAME PARAMETERS AND STABILITY MARGINS}

In this section, we judge the system's stability by looking at the closed-loop poles when the proposed state feedback controller is used. Differences in chosen game parameters produce a change in the closed-loop pole locations with the optimal controller.

In figure 3, the first plot has the attacker with half the control cost of the defenders $\left(r_{22}=0.5\right)$ i.e. the attacker's input is half as expensive as that of the other cars. This is equivalent to saying the attacker can apply double the control input of the regular cars. We see that by choosing just the negative of standard parameters $\left(q_{3}=-1, q_{8}=-2\right)$, we get the first pole pair in the first plot, which are very well damped. As we vary more towards instability, we see that $q_{8}$ changes sign before it gets close to the imaginary axis.

In the next plot, where $q_{3}$ is varied, $q_{8}$ had to be zero in order to produce any instability at all. However, when it was set to zero, instability is quickly brought on.

In the final plot, $r_{22}$ is varied to make the attacker's control almost twenty times as cheap as the defenders'. Only then does instability ensue. Again this is an unrealistic scenario and we see that just by applying more control input, we do not achieve an optimal attack or degradation instability.

It is worth mentioning at this point that these parameters are not varied by the attacker. They are in fact means to set up a situation in which to find the optimal control law. Another way of saying this is that if these parameters are set and then the attacker tries to cause oscillations or collisions in the platoon by choosing some unstable gains, it will not be an optimal saddle-point solution. Hence the attacker will end up losing out in terms of the game itself.

The purpose of this section was to illustrate that a game would have to be set up dramatically different from any usual setup to produce a situation where the attacker would benefit by causing instability. The following section shows two sets of simulation data which also highlight one more aspect of the pole locations: their frequency.
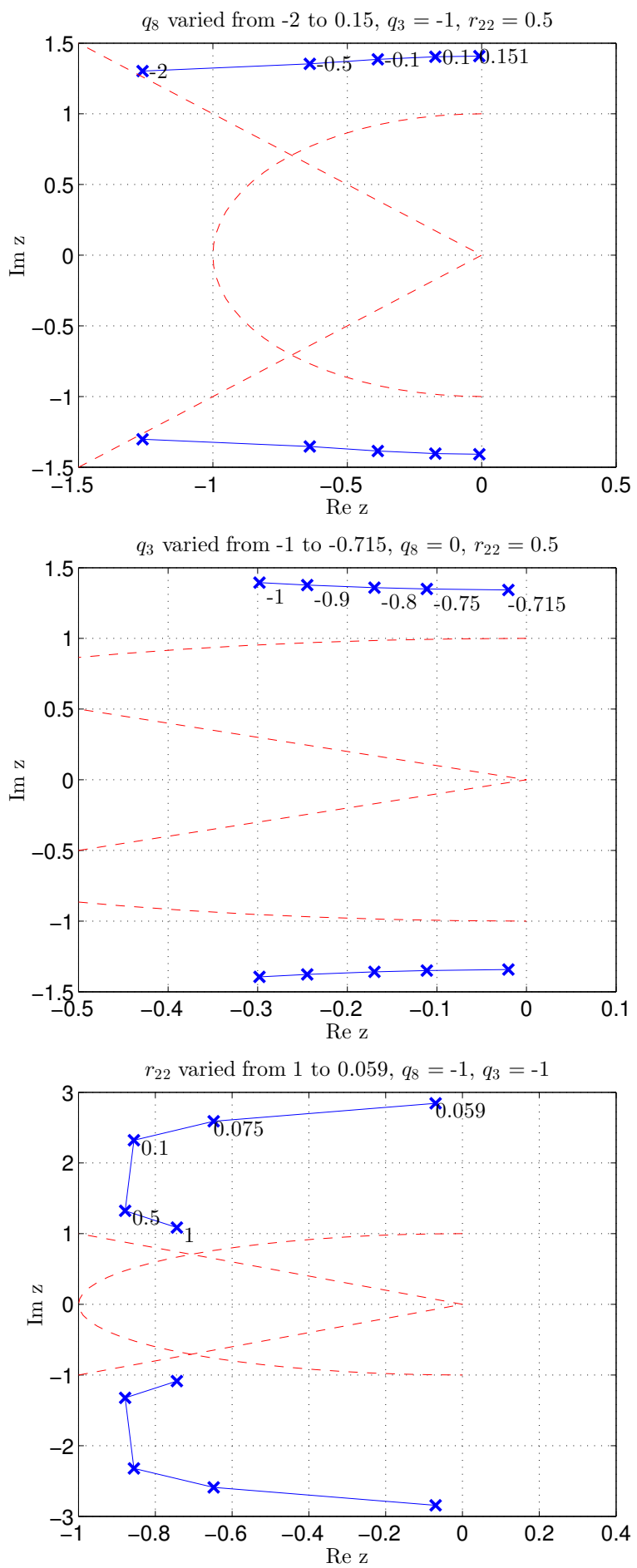

Figure 3: Least damped pole locations change based on parameter varied. Unit circle for $1 \mathrm{rad} \mathrm{s}^{-1}$ and $45^{\circ}$ lines shown in dashed red. Parameters were changed till the least stable poles moved towards the right-half plane and were close to being unstable. 


\section{SIMULATION RESULTS}

For the simulation results presented here, the split level controller approach from [22] is explicitly incorporated. The low level controller realizes the acceleration command by compensating for the effects of inertia, drag and air resistance. But this low level control signal is saturated as a real motor would be, which imparts a top speed $v_{\max }$ and maximum acceleration $a_{\max }$ to the model. While in saturation, the model does not conform to a linear quadratic game (as will be shown in the results) but is necessary for a more realistic test.

The data in table 1 were used for the subsequent simulations. There were 5 cars in total and the attacker was at position 3.

\begin{tabular}{|l|l|}
\hline Parameter & Value \\
\hline$\sigma_{\text {ref }}$ & $9 \mathrm{~m}$ \\
$l$ & $4.5 \mathrm{~m}$ \\
$\dot{x}_{\text {ref }}$ & $25 \mathrm{~m} \mathrm{~s}^{-1}$ \\
$v_{\max }$ & $50 \mathrm{~m} \mathrm{~s}^{-1}$ \\
$a_{\max }$ & $5 \mathrm{~m} \mathrm{~s}^{-2}$ \\
\hline
\end{tabular}

Table 1: Platooning data used in the simulations.

Since the overshoot and damping characteristics need to be visualized, the cars were started with their desired separations but with an initial velocity of zero.

In figure 4, it is observed that the nominal parameters we started off with produce a stable solution as expected. The cars speed up from zero and catch up to the reference trajectory. Notice that at the start (since they get an instantaneous command to speed up to $25 \mathrm{~m} \mathrm{~s}^{-1}$ ) the accelerations saturate and reduce as the speed increases. This is a nonlinear effect of using a realistic car model.

In figure 5, we see that the given choice of parameters does produce some oscillatory behavior. However note that the amplitude is not nearly enough to cause any car to collide into another, as none of the separations become less than car length. The reason is simply that most physical systems (like our cars) act as low pass filters and in order to produce a higher amplitude, one needs a lower frequency.

At this point we refer the reader back to figure 3 and point out that these poles are not at low frequencies. At least in all the cases shown, the poles do not move towards the area around the origin when varying parameters. This is another observed property that low-frequency, high-swinging solutions are not optimal in the game theoretic setups we analyzed.

\section{DISCUSSION}

In this section we highlight some of the key aspects of this gametheoretic framework and also some limitations to the current approach of solving a Riccati equation.

Since this approach is similar in appearance and in essence to the LQR, it seems pertinent to ask whether this approach can offer some of the same benefits as its optimal control counterpart. The LQR offers a bounded phase margin, which automatically guarantees stability and convergence, and results exist pertaining to its robustness characteristics [15]. In figure 5, oscillations were observed, which points to a low phase margin. The question of
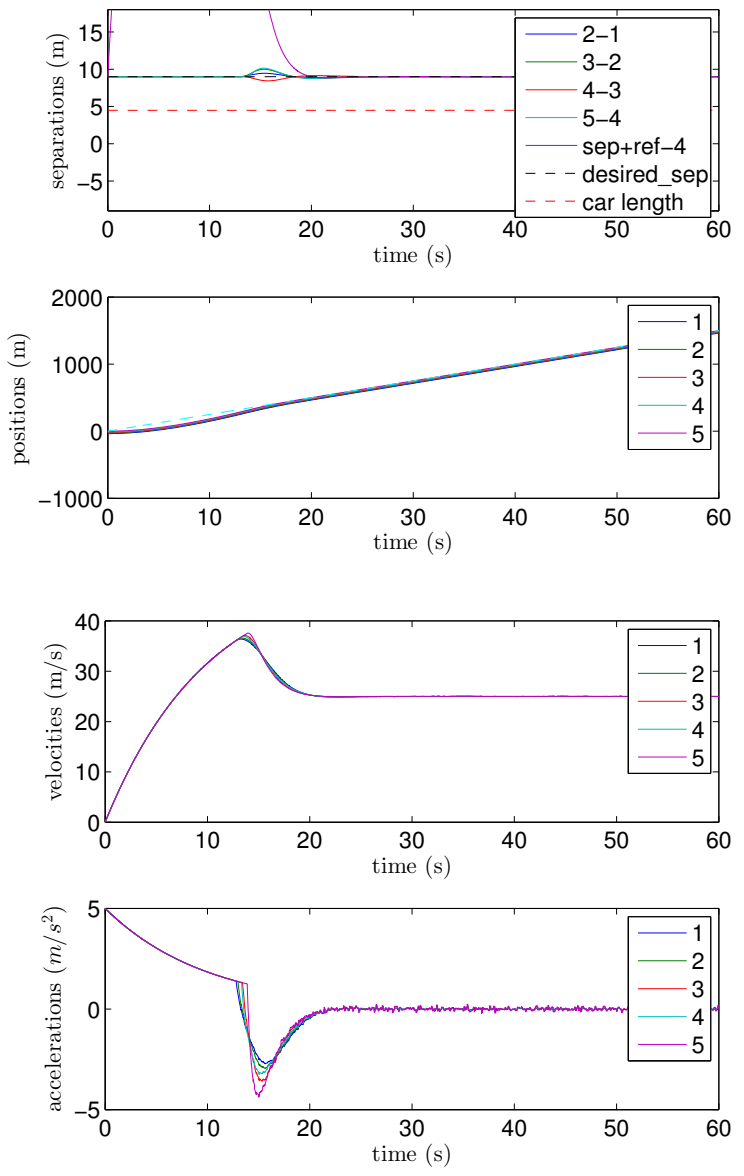

Figure 4: Simulation results of game theoretic solution $\left(q_{3}=\right.$ $\left.-1, q_{8}=-2, r_{22}=0.5\right)$. These parameters are ones that one would realistically set.

whether there are conditions on $Q$ and $R$ under which the system is guaranteed to be stable is valid and, like the LQR, an analytical proof of this might be possible.

It should be noted that forming a proper game-theoretic problem requires one or two conditions to be met. Just as in optimal control, the $R_{i i}$ matrices that weight the player's own control inputs have to be positive definite. This means that it can never benefit a player to apply ever increasing control input. However the $Q$ matrices as we have seen already, can have a mix of nonnegative and negative eigenvalues. This aspect of the solution makes it interesting to ask the question: when do saddle-point solutions exist. [4] treats this question and derives some necessary and sufficient conditions, albeit with some limitations, for having a valid solution.

For our system, we solved a single Riccati equation which emerges in the zero-sum case. If we were to allow nonzero-sum formulations, we would need to solve a system of Riccati equations simultaneously [28]. The computational tools we used were build primarily for the purposes of standard optimal control and thus only allowed unique stabilizing solutions to a single Riccati equation. As this 

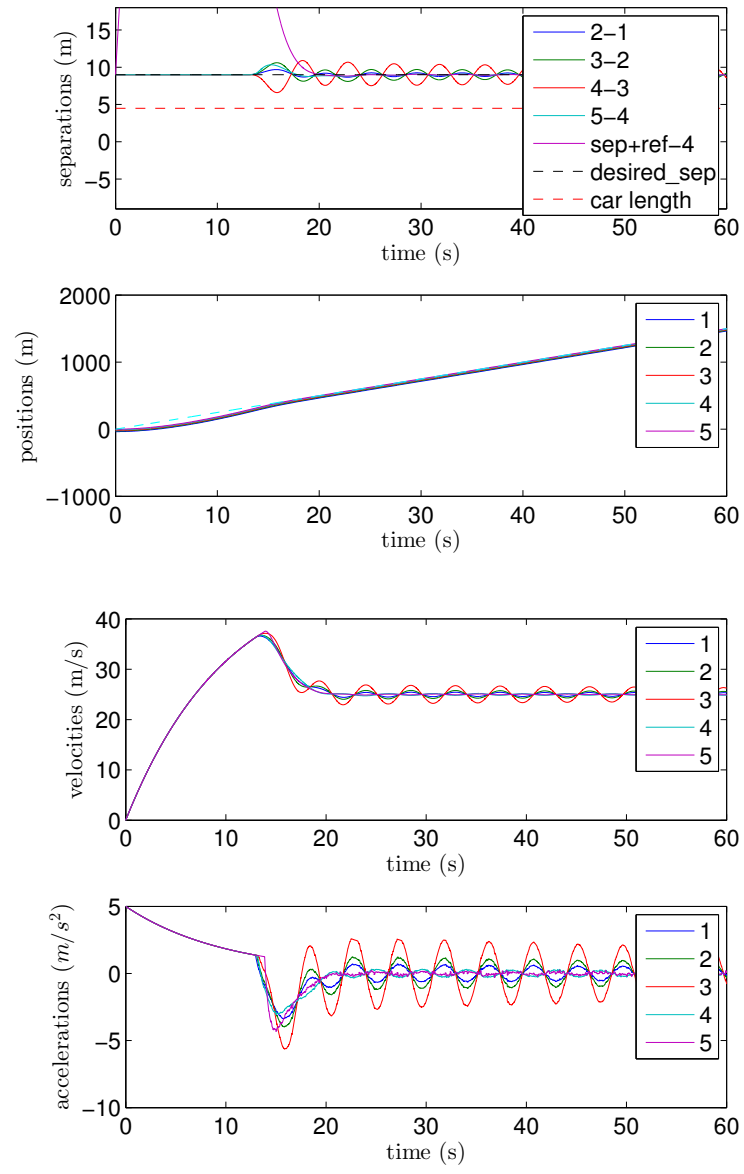

Figure 5: Simulation results of game theoretic solution $\left(q_{3}=\right.$ $\left.-1, q_{8}=0.151, r_{22}=0.5\right)$. These parameters are ones that would yield a near oscillatory solution. First plot shows that no separation ever gets low enough to cause a collision (does not cross the dashed red line).

equation can normally have more than one solution, but at maximum only one stabilizing one, it might be worthwhile, in order to entertain the unstable case, to ask which unstable solution actually corresponds to the saddle-point or Nash equilibrium point in a game-theoretic formulation.

Furthermore, this setup can be generally extended to a higher number of cars and multiple attackers (who would still form the same 'player'). Those results might enable the sort of attacks we might have liked to see here, but varying those parameters would add another dimension of complexity to an already complex system. We are aware that the results presented here are not entirely exhaustive and that the conclusions we would like to draw from these results might be overturned by a single case where the attack does cause the desired behavior. However, these results are similar to those provided by optimal control theory and perhaps some general statements can be made on the nature of solutions provided by game theory in terms of system performance such as a guaranteed phase margin.

Alternatively, a stochastic technique such as Monte Carlo runs might be employed while varying parameters such as the $Q$ and $R$ matrices, number of cars and attacker position. Further study would be required to construct an appropriate parametrization of these variables based on system properties. At the very least, it might provide a range of suitable values where the attacker does not benefit from causing collisions and these could be cross correlated with admissible parameters.

\section{CONCLUSION}

This paper demonstrates a game-theoretic framework to the problem of vehicular platooning. An infinite time horizon, linear quadratic game was formulated whose saddle point solution was found using methods from optimal control. The various solutions to this problem demonstrated that it is hard to achieve a setting (with the above qualities) that sees the attacker colliding with other cars or causing large oscillations in traffic flow while still fairing well in the game itself; it is usually not in the best interest of the attacker to disrupt regular operation. Further extensions of this work can involve solving the nonzero sum case and possibly entertaining unstable solutions. The effect of collusion by multiple attackers can also be considered in future work.

\section{ACKNOWLEDGMENTS}

This work is supported by the National Science Foundation under Grant No. 1410000.

\section{REFERENCES}

[1] 2002. The SARTRE project. (2002). www.sartre-project.net [Online; accessed 15-June-2015].

[2] P. Barooah and J.P. Hespanha. 2005. Error Amplification and Disturbance Propagation in Vehicle Strings with Decentralized Linear Control. In Decision and Control, 2005 and 2005 European Control Conference. CDC-ECC '05. 44th IEEE Conference on. 4964-4969. DOI : http://dx.doi.org/10.1109/CDC.2005.1582948

[3] T. Basar. 1991. A dynamic games approach to controller design: disturbance rejection in discrete-time. IEEE Trans. Automat. Control 36, 8 (Aug 1991), 936-952. DOI : http://dx.doi.org/10.1109/9.133187

[4] Tamer Basar and Geert Jan Olsder. 1995. Dynamic noncooperative game theory. Academic Press, London, San Diego. http://opac.inria.fr/record=b1090171

[5] RJ Caudill and WL Garrard. 1977. Vehicle-follower longitudinal control for automated transit vehicles. Fournal of Dynamic Systems, Measurement, and Control 99, 4 (1977), 241-248.

[6] Yuan Chen, Soummya Kar, and José MF Moura. 2016. Cyber physical attacks constrained by control objectives. In 2016 American Control Conference (ACC). IEEE, 1185-1190.

[7] Soodeh Dadras, Ryan M Gerdes, and Rajnikant Sharma. 2015. Vehicular Platooning in an Adversarial Environment. In Proceedings of the 10th ACM Symposium on Information, Computer and Communications Security. ACM, 167-178.

[8] Daniel D. Dunn. 2015. ATTACKER-INDUCED TRAFFIC FLOW INSTABILITY IN A STREAM OF AUTOMATED VEHICLES. Master's thesis. Utah State University, Logan, Utah.

[9] Ather Gattami, Assad Al Alam, Karl H. Johansson, and Claire J. Tomlin. 2011. Establishing Safety for Heavy Duty Vehicle Platooning: A Game Theoretical Approach. \{IFAC $\}$ Proceedings Volumes 44, 1 (2011), 3818 - 3823. DOI : http: //dx.doi.org/10.3182/20110828-6-IT-1002.02071 18th \{IFAC $\}$ World Congress.

[10] Ryan M Gerdes, Chris Winstead, and Kevin Heaslip. 2013. CPS: an efficiencymotivated attack against autonomous vehicular transportation. In Proceedings of the 29th Annual Computer Security Applications Conference. ACM, 99-108.

[11] D. Grimsman, V. Chetty, N. Woodbury, E. Vaziripour, S. Roy, D. Zappala, and S. Warnick. 2016. A case study of a systematic attack design method for critical infrastructure cyber-physical systems. In 2016 American Control Conference (ACC). 296-301. DOI : http://dx.doi.org/10.1109/ACC.2016.7524931

[12] Zhu Han. 2012. Game theory in wireless and communication networks: theory, models, and applications. Cambridge University Press. 
[13] Rufus Isaacs. 1999. Differential games: a mathematical theory with applications to warfare and pursuit, control and optimization. Courier Corporation.

[14] J. Lavaei. 2012. Decentralized Implementation of Centralized Controllers for Interconnected Systems. IEEE Trans. Automat. Control 57, 7 (July 2012), 18601865. DOI : http://dx.doi.org/10.1109/TAC.2011.2180089

[15] E. Lavretsky and K. Wise. 2012. Robust and Adaptive Control: With Aerospace Applications. Springer London. https://books.google.com/books?id=a2128lhlWfQC

[16] Kuo-Yun Liang, J. Martensson, and K.H. Johansson. 2014. Fuel-saving potentials of platooning evaluated through sparse heavy-duty vehicle position data. In Intelligent Vehicles Symposium Proceedings, 2014 IEEE. 1061-1068. DOI:http: //dx.doi.org/10.1109/IVS.2014.6856540

[17] Yilin Mo, Emanuele Garone, Alessandro Casavola, and Bruno Sinopoli. 2010. False data injection attacks against state estimation in wireless sensor networks. In Decision and Control (CDC), 2010 49th IEEE Conference on. IEEE, 5967-5972.

[18] John Nash. 1951. Non-Cooperative Games. Annals of Mathematics 54, 2 (1951), 286-295. http://www.jstor.org/stable/1969529

[19] Esha D Nerurkar, Stergios I Roumeliotis, and Agostino Martinelli. 2009. Distributed maximum a posteriori estimation for multi-robot cooperative localization. In Robotics and Automation, 2009. ICRA'09. IEEE International Conference on IEEE, 1402-1409.

[20] Fabio Pasqualetti, R. Carli, A. Bicchi, and F. Bullo. 2010. Identifying cyber attacks via local model information. In Decision and Control (CDC), 2010 49th IEEE Conference on. 5961-5966. DOI : http://dx.doi.org/10.1109/CDC.2010.5717914

[21] L. Peppard. 1974. String stability of relative-motion PID vehicle control systems. Automatic Control, IEEE Transactions on 19, 5 (Oct 1974), 579-581. DOI : http: //dx.doi.org/10.1109/TAC.1974.1100652

[22] R. Rajamani. 2011. Vehicle Dynamics and Control. Springer. https://books.google. com/books?id=eoy19aWAjBgC

[23] Bhaskar Ramasubramanian, MA Rajan, and M Girish Chandra. 2016. Structural resilience of cyberphysical systems under attack. In 2016 American Control
Conference (ACC). IEEE, 283-289.

[24] W. Ren and D. Green. 1994. Continuous platooning: a new evolutionary operating concept for automated highway systems. In American Control Conference, 1994, Vol. 1. 21-25 vol.1. DOI : http://dx.doi.org/10.1109/ACC.1994.751685

[25] Tom Robinson, Eric Chan, and Erik Coelingh. 2010. Operating platoons on public motorways: An introduction to the sartre platooning programme. In 17th world congress on intelligent transport systems, Vol. 1. 12.

[26] Rajnikant Sharma, Randy W Beard, Clark N Taylor, and Stephen Quebe. 2012. Graph-based observability analysis of bearing-only cooperative localization. IEEE Transactions on Robotics 28, 2 (2012), 522-529.

[27] S. Sheikholeslam and C.A. Desoer. 1993. Longitudinal control of a platoon of vehicles with no communication of lead vehicle information: a system level study. Vehicular Technology, IEEE Transactions on 42, 4 (Nov 1993), 546-554. DOI : http://dx.doi.org/10.1109/25.260756

[28] A. W. Starr and Y. C. Ho. 1969. Nonzero-sum differential games. Fournal of Optimization Theory and Applications 3, 3 (1969), 184-206. DOI : http://dx.doi. org/10.1007/BF00929443

[29] D. Swaroop and J.K. Hedrick. 1996. String stability of interconnected systems. Automatic Control, IEEE Transactions on 41, 3 (Mar 1996), 349-357. DOI : http: //dx.doi.org/10.1109/9.486636

[30] C. J. Tomlin, J. Lygeros, and S. Shankar Sastry. 2000. A game theoretic approach to controller design for hybrid systems. Proc. IEEE 88, 7 (July 2000), 949-970. DOI : http://dx.doi.org/10.1109/5.871303

[31] L. Xiao and F. Gao. 2011. Practical String Stability of Platoon of Adaptive Cruise Control Vehicles. IEEE Transactions on Intelligent Transportation Systems 12, 4 (Dec 2011), 1184-1194. DOI : http://dx.doi.org/10.1109/TITS.2011.2143407

[32] Diana Yanakiev and Ioannis Kanellakopoulos. 1996. A simplified framework for string stability analysis in AHS. In Proceedings of the 13th IFAC World Congress. 177-182. 